\title{
Impact du Dividende Demographique sur la Croissance Economique au Senegal
}

\author{
Latif Dramani \\ Centre de Recherche en Economie et Finance Appliquées de Thiès \\ CREFATI/Université de Thiès \\ latif.dramani@gmail.com
}

\section{Résumé}

L'impact de la démographie sur la croissance économique a fait l'objet de plusieurs études, mais cellesci se limitent très souvent au rôle de la taille et de la croissance de la population, et négligent les effets de la structure par âge sur les performances économiques. Pourtant, étant donné que les individus présentent des comportements économiques qui varient selon les âges, toute modification de la structure par âge d'une population a des implications sur les variables économiques. L'objectif de cet article est d'examiner à travers la théorie de l'économie générationnelle la relation entre l'évolution de la structure par âge et la croissance économique au Sénégal. Les résultats de l'estimation à partir d'un modèle à correction d'erreur montrent qu'une augmentation du ratio de soutien d'un point de pourcentage se traduit par une hausse du revenu par tête de 3,7\%. Le dividende démographique s'avère donc être une source de croissance pour l'économie sénégalaise.

Mots clés: Dividende démographique, fécondité, croissance économique, ratio de soutien, Sénégal.

Codes JEL: EOI, E2I, II8, JI3, O55.

\begin{abstract}
Several studies have examined the impact of demographics on economic growth through the effects of the size and growth of the population, and overlook the effects of the changing age structure on economic performance. However, since individuals have economic behaviors that vary according to age, any change in the age structure of a population has implications on economic variables. The purpose of this paper is to examine the relationship between changing population age structure and economic growth in Senegal. The error correction model estimation shows that an increase of one percentage point in the support ratio is associated with an increase in per capita income of 3.7 percentage. The demographic dividend proves to be a source of growth for the Senegalese economy.
\end{abstract}

Keywords: Demographic Dividend, Fertility, Economic Growth, Support Ratio, Senegal

JEL Classification: E0I, E2I, II8, JI3, O55.

\footnotetext{
' Centre de Recherche en Economie et Finance Appliquées de Thiès.
} 


\section{Introduction}

L'étude du rôle des facteurs démographiques dans l'analyse des sources de la croissance économique s'est longtemps limitée aux effets de la taille et de la croissance de la population. Ce débat a négligé l'importance de la structure par âge et de la dynamique de la population sur les performances économiques. Les changements de la structure par âge sont toutefois importants car, à en croire Bloom et al. (200I), la population présente des comportements économiques différents suivant les groupes d'âge. La transition démographique en cours dans plusieurs pays en développement mais achevée dans la plupart des pays développés, peut être décrite comme une évolution de la structure par âge de la population. En effet, la transition démographique est un processus au cours duquel un pays passe des taux de fécondité et de mortalité élevés à des taux de fécondité et de mortalité faibles. Au cours de ce processus, la population en âge de travailler augmente temporairement plus vite le nombre de personnes jeunes et âgées.

Cette modification de la structure par âge a des implications sur les grandeurs économiques du pays. En effet, comme les personnes en âge de travailler sont plus productives que celles qui sont en dehors de ce groupe d'âge, une augmentation de leur proportion contribue à des taux d'épargne plus élevés, et donc permet de libérer des ressources pour l'investissement dans le développement économique et le bien-être des familles. $\mathrm{Ce}$ mécanisme se nomme "dividende démographique ".

A l'instar de nombreux pays d'Afrique subsaharienne, le Sénégal a amorcé la transition démographique depuis quelques années avec un taux de fécondité qui est en baisse (Adjamagbo et Antoine (2002); Pison et al. (1997)). Bien qu'elle soit encore lente, cette baisse est susceptible de modifier la structure par âge de la population. Au vu de cette situation, il est opportun de se demander si le pays pourrait tirer profit de ce changement de la structure par âge.

La présente étude analyse cette piste de recherche insuffisamment explorée dans la littérature économique pour le cas Sénégal. II s'agit de rechercher l'existence d'une relation entre l'évolution de la structure par âge et la croissance économique. Plus précisément, l'étude permet d'examiner si le dividende démographique contribue de façon positive à l'augmentation du revenu par tête.

Le reste du document est organisé en cinq sections. La première section relate les faits stylisés marquants du Sénégal sur le plan économique et démographique. La seconde section, qui fait l'objet de la revue de la littérature, présente les principaux résultats des recherches portant sur le lien entre les facteurs démographiques et la croissance économique. $\mathrm{La}$ troisième section présente l'approche méthodologique adoptée dans l'analyse de l'impact du dividende démographique sur la croissance économique du Sénégal. Les résultats issus de l'analyse économétrique ainsi que leurs interprétations font l'objet de la quatrième et cinquième section respectivement.

\section{FAITS STYLISES}

Tendances démographiques du Sénégal: une transition en cours

\section{Un accroissement important de la population}

Au moment de son accession à l'indépendance en 1960, le Sénégal avait une population estimée à 3,5 millions d'habitants. Seize années plus tard, la population est passée à 5 millions selon le recensement de 1976, soit un accroissement moyen annuel de $2,4 \%$.

Les recensements de 1988, 2002 et 2013 l'ont estimée respectivement à 6,$9 ; 9,9$ et 13,5 millions d'habitants. Selon les projections démographiques récentes, cette population pourrait atteindre 19,3 millions en 2025 et 25,7 millions en 2035 (ANSD, 20I5). Le taux d'accroissement intercensitaire est de 2,7\% par an entre 1976 et 1988. Ce taux a légèrement baissé pour se situer à 2,5\% par an entre 1988 et 2013. Cela correspond donc à un doublement de la population tous les 25 ans.

\section{Une mortalité des enfants en baisse}

Le Sénégal enregistre une baisse de la mortalité des enfants depuis les années 1960. Selon les enquêtes démographiques et de santé (EDS), la mortalité infanto-juvénile a été réduite de près de la moitié entre 1963 et 1997 en passant de $287 \%$ ò $139 \%$ o. On observe surtout que la tendance à la baisse de la mortalité des enfants est rapide à partir de la fin des années 1970. Le taux de mortalité infanto-juvénile a en effet chuté de $53 \%$ ontre 1978 et 1986, soit une baisse annuelle moyenne de $7 \%$.

Les données les plus récentes révèlent que la baisse de la mortalité non seulement se poursuit jusqu'à ce jour, mais surtout elle s'est accentuée au cours ces dernières années. En effet, selon les données de l'EDS de 2005 et EDS-MICS ${ }^{2}$ de 20I0$\mathrm{II}$, le taux de mortalité infantile est passé de $68 \%$ o en 1997 à $61 \%$ on 2005, avant de chuter drastiquement $47 \%$ on 2010-20II. De plus, la baisse est très importante pour la mortalité infantojuvénile qui a chuté de la moitié (de 139\%o en 1997 à

\footnotetext{
2 EDS - MICS: Enquête démographique et de santé à indicateurs multiples au Sénégal
} 
$72 \%$ en $2010-2011$ ), mais surtout pour la mortalité juvénile dont le taux a été divisé par 3 en l'espace de treize années (77\%o en I 997 à $26 \%$ on 20 I0-20 I I).

La baisse de la mortalité des enfants, principal indicateur de l'état de santé d'une population, s'explique par la multiplication des programmes de santé, en particulier de vaccination, l'amélioration des infrastructures sanitaires, de la connaissance des conditions d'hygiène et de l'amélioration de l'état nutritionnel des enfants, etc. (Adjamagbo et Antoine, 2002). Entre également en ligne de compte le niveau d'instruction des mères. En effet, les données de I'EDS III (I997), EDS IV (2005) et EDS-MICS (20I020II) révèlent que les enfants dont la mère a un niveau d'éducation primaire ou secondaire ont respectivement deux ou trois fois plus de chance d'atteindre leur cinquième anniversaire que ceux dont la mère est non instruite. En effet selon ces données, le taux de mortalité infanto-juvénile est de $96 \%$ et $55 \%$ pour les mères de niveau d'études primaire et secondaire respectivement, contre 154\%० pour les mères non instruites en 1997; $94 \%$ et $60 \%$ o contre $152 \%$ en 2005 ; $63 \%$ et $36 \%$ o contre $97 \%$ o en 201 I.

Toutefois, il est important de noter qu'à partir de 2005, la baisse de la mortalité est très significative audelà du premier anniversaire, ceci quel que soit le niveau d'instruction de la mère. En effet, entre 2005 et $20 \mathrm{II}$, on enregistre chez les mères n'ayant aucun niveau d'études, une baisse de la mortalité juvénile et infanto-juvénile de près de la moitié et du tiers respectivement. Sur la même période, la mortalité des enfants de moins 5 ans a baissé aussi de près de la moitié chez les mères ayant un niveau d'études secondaire ou plus.

Par ailleurs, on observe des disparités parfois énormes entre les milieux de résidence surtout concernant le risque de décéder avant le cinquième anniversaire. En effet, la mortalité des enfants de moins de 5 ans est plus importante en milieu rural qu'en milieu urbain; l'écart des taux de mortalité infanto-juvénile est de 82\%o en 1992, 69\%० en 2005 et $40 \%$ en $20 \mathrm{Il}$. Toutefois, la tendance de la baisse des taux est relativement est la même pour les deux milieux, bien qu'elle soit légèrement plus marquée en milieu urbain (Source: Données EDS I, II, III, IV et EDS-MICS 20I0-II).

\section{Une fécondité en baisse également}

Au cours des vingt premières années d'indépendance, le Sénégal connaissait une fécondité élevée, l'enquête sénégalaise de fécondité (ESF, 1978) estimait à 7,1 enfants par femme l'indice synthétique de fécondité (ISF) qui mesure le nombre moyen d'enfants par femme à la fin de sa vie reproductive. Par la suite, la comparaison avec l'enquête démographique et de santé de 1986 (EDS, 1986) indique une légère baisse de la fécondité: I'ISF est passé à 6,6 enfants par femme en 1986. Les prochaines enquêtes démographiques de santé ont confirmé cette tendance à la baisse sur les périodes suivantes où la fécondité décroît à peu près au même rythme. Ainsi, en 1992 et 1997, les femmes ont en moyenne 6,0 et 5,7 enfants respectivement. Les dernières enquêtes révèlent que le nombre de naissances vivantes par femme a chuté à 5,3 en 2005 , puis à 5,0 en 2010 2011 .

La baisse de la fécondité observée présentement suit une baisse de la mortalité qui, elle autre, a commencé un peu plus tôt (fin des années 1970); cela révèle que le Sénégal est entré dans la deuxième phase de la transition démographique.

\section{Fécondité et milieu de résidence}

Une des particularités de la baisse de la fécondité au Sénégal est qu'elle s'étend difficilement au milieu rural, alors qu'elle est déjà bien amorcée dans les villes. En effet, la baisse de la fécondité dans les villes sénégalaises est relativement ancienne: le nombre moyen d'enfants par femme passe de 6,6 en 1978 à 5,5 en 1986 en milieu urbain. Durant la même période, I'ISF observé en milieu rural bouge très faiblement: 7,5 à 7,I enfants. La baisse est également faible en 1992, année au cours de laquelle l'ISF est de 6,7 enfants par femme. Les enquêtes plus récentes confirment que les changements de comportement en matière de baisse de la fécondité sont plus lents dans les zones rurales que ce qu'on observe en zones urbaines (voir tableau I ci-dessous).

Tableau I: Evolution de I'ISF selon le niveau d'études et le milieu de résidence, 1978-20 I I

\begin{tabular}{lllllll}
\hline Niveau d'études & $\mathbf{I 9 7 8}$ & $\mathbf{1 9 8 6}$ & $\mathbf{1 9 9 2}$ & $\mathbf{1 9 9 7}$ & $\mathbf{2 0 0 5}$ & $\mathbf{2 0 I 0 - I I}$ \\
\hline Aucun & 7,4 & 6,8 & 6,5 & 6,3 & 6,1 & 5,8 \\
Primaire & $7, \mathrm{I}$ & 5,2 & 5,7 & 5,2 & 4,8 & 4,5 \\
Secondaire ou + & 3,6 & 3,7 & 3,8 & 3,1 & 3,0 & 2,9 \\
\hline Milieu de résidence & & & & & & \\
\hline Urbain & 6,6 & 5,4 & 5,1 & 4,3 & 4,1 & 3,9 \\
Rural & 7,5 & 7,1 & 6,7 & 6,7 & 6,4 & 6,0 \\
\hline
\end{tabular}




\begin{tabular}{lllllll}
\hline Ensemble & 7,2 & 6,6 & 6,0 & 5,7 & 5,3 & 5,0 \\
\hline
\end{tabular}

Source: ESF 1978; EDS I, II, III, IV; EDS-MICS 2010-II

En revanche dans les zones urbaines, une fois enclenchée, la baisse de la fécondité est plus rapide que les zones rurales. En effet, sur la période allant de 1978 à 20 I0-II, le nombre moyen d'enfants par femme est passé de 6,6 à 3,9. La fécondité a ainsi diminué de 2,7 enfants dans les villes alors qu'elle n'a baissé que de I,5 enfant en milieu rural sur la même période. L'écart entre les milieux urbain et rural est donc important en matière de fécondité.

\section{Fécondité et niveau d'études}

A l'instar des autres pays africains, on observe l'existence d'un lien entre la fécondité et le niveau d'études au Sénégal. En effet, comme le montre le tableau I ci-avant, le niveau de fécondité est nettement plus bas parmi les femmes ayant atteint le niveau d'études secondaire que parmi les femmes moins éduquées. En 2010-20II par exemple, I'ISF passe de 5,8 enfants par femme sans instruction à 4,5 enfants pour la femme de niveau primaire et baisse à 2,9 enfants pour la femme ayant atteint le niveau secondaire ou plus. Cela révèle donc que plus le niveau d'études de la femme est élevé, plus faible est le nombre moyen d'enfants de celle-ci. Autrement dit, plus les femmes restent longtemps à l'école, moins elles font d'enfants.

Ainsi, les différences de relations entre l'ISF selon le niveau d'éducation permettent de penser que les femmes les plus éduquées se marient plus tard, utilisent davantage la contraception, ont une meilleure connaissance des méthodes contraceptives et de leurs accès, disposent d'une autonomie supérieure en matière de décisions reproductives et sont plus enclines à utiliser une contraception compte tenu des coûts d'opportunité élevés des grossesses non désirées.

Par ailleurs, le niveau relativement bas de la fécondité dans les villes observé dans le tableau I peut s'expliquer en partie par les conditions de l'offre scolaire en milieu urbain qui plus favorisé que le milieu rural.
Structure par âge de la population: ratio de dépendance démographique en baisse

L'examen de la structure de la population sénégalaise révèle une population jeune. En effet, au recensement de 2013, 42\% des Sénégalais sont âgés de moins 15 ans, et la moitié de la population a moins de 18 ans. Les personnes du troisième âge représentent $3,5 \%$ et les personnes âgées de 15 à 64 ans sont estimées à $56 \%$. C'est dire que le coefficient de dépendance est élevé. Les calculs effectués à partir des données du RGPHAE ${ }^{3}$ montrent que 83,7 personnes potentiellement inactives (moins de 15 ans et 65 ans et plus) sont prises en charge par 100 personnes potentiellement actives (15 à 64 ans). Toutefois, malgré ce niveau élevé, le taux de dépendance en 2013 est plus faible que celui des années précédentes. En effet, ce taux qui était de 100 actifs pour 103,3 inactifs et 86,5 inactifs respectivement en 1988 et en 2002 , est donc en baisse et pourrait atteindre, selon les projections de l'ANSD (2015), 79 pour 100 en 2035.
${ }^{3}$ Recensement Général de la Population, de l'Habitat, de l'Agriculture et de l'Elevage. 
Graphique I: Ratio de dépendance démographique, 1988-2013

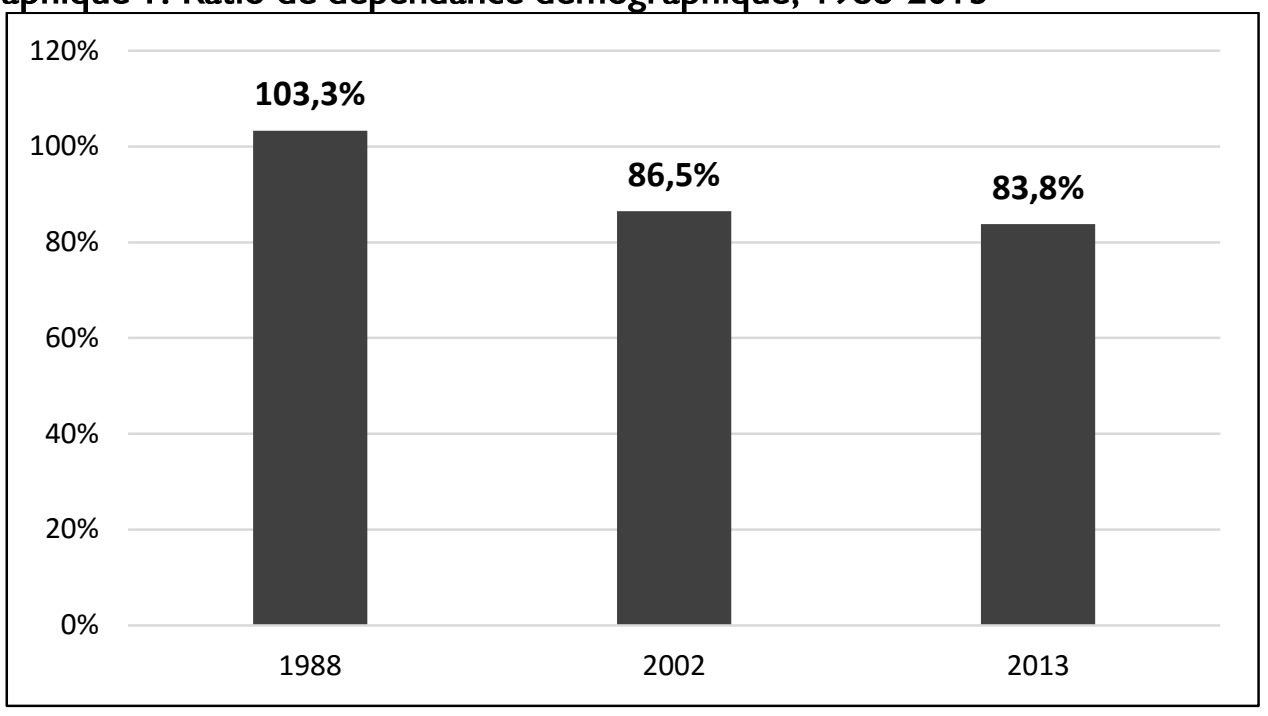

Sources : RGPHAE 2013 et RGPH 2002.

Situation économique du Sénégal: une croissance qui peine à décoller

Au cours des 20 premières années qui ont suivi son accession à la souveraineté internationale, le Sénégal a enregistré des performances économiques peu satisfaisantes avec une croissance moyenne annuelle de $2,2 \%$ de la production pour une croissance démographique estimée à $2,7 \%$ par an, soit une baisse de $0,2 \%$ du PIB par habitant. La dégradation de la situation économique du pays a poussé l'État vers des endettements colossaux. Le service de la dette extérieure représentait le tiers des exportations et le déficit extérieur courant représentait $14,5 \%$ du PIB.

Pour assainir l'environnement économique et réduire les dépenses publiques, les politiques d'ajustement structurel (PAS) ont été mises en œuvre dans les années 1980 avec le soutien des institutions de Bretton Woods. Ces politiques avaient pour but de restructurer le système bancaire et les entreprises publiques et de développer l'initiative privée. Les conséquences des PAS ont été désastreuses avec environ 20.000 pertes d'emploi et une progression continue du chômage et de la pauvreté (Dramani, à paraitre).

Comme tous les autres pays de la zone franc, le Sénégal perdait de plus en plus sa compétitivité sur le plan mondial conduisant en 1994 à une dévaluation de $50 \%$ du franc CFA en monnaie étrangère. La dévaluation du franc CFA accompagnée d'une tenue assez rigoureuse des finances publiques a permis d'observer une expansion économique plus soutenue et un peu plus vigoureuse que par le passé, avec un taux de croissance du PIB de l'ordre de $5 \%$ par an en moyenne (Diagne et Daffé, 2002). Ainsi, pour la première fois dans l'histoire économique du Sénégal indépendant, le taux de croissance du PIB par tête a ainsi pu se maintenir à plus de $2 \%$ par an en moyenne pendant une dizaine d'années successives.

Cependant, cette nouvelle relance économique n'a pas eu d'effets significatifs sur l'emploi, encore moins permettre de compenser le recul observé dans le passé. En effet, la paurreté y demeure toujours élevée quand bien même elle a baissé, en passant de de $61,2 \%$ en $1994-1995$ à $55,2 \%$ en $2001-2002$ (ESAM I et 2$)^{4}$.

Les performances économiques des années les plus récentes sont tout aussi décevantes. Depuis 2006, l'économie sénégalaise est caractérisée par une croissance relativement faible. Le taux de croissance du PIB est de 3,3\% en moyenne par année entre 2006 et 2013 (Source: Comptes nationaux, ANSD), et se situe en deçà de celui de l'ensemble de l'Afrique subsaharienne qui s'établit, selon la Banque mondiale, à $6 \%$ en moyenne par année sur la même période. Compte tenu d'un taux d'accroissement démographique annuel de $2,5 \%$, le taux de croissance économique observé sur la période n'est pas assez consistant pour faire reculer la pauvreté de façon significative (Banque Mondiale, 20I4). C'est en partie l'une des raisons qui expliquent, selon la Banque Mondiale (2014) le fait que l'incidence de pauvreté n'a baissé que I,6 point entre 2005 et $201 \mathrm{I}$ (elle passe de $48,3 \%$ en $2005-2006$ à $46,7 \%$ en $20 \mathrm{II}$ ), tandis que le nombre absolu de pauvres ne cesse d'augmenter pour atteindre 6,2 millions en 2011 .

\footnotetext{
${ }^{4}$ ESAM : Enquête sénégalaise auprès des ménages.
} http://aps.journal.ac.za 


\section{BREVE REVUE DE LITTERATURE}

La relation entre population et croissance économique est un sujet largement abordé dans la littérature économique. Le débat porte sur les effets de la croissance de la population sur le développement économique ou, plus précisément, il est question de savoir si la croissance démographique favorise ou réduit ou est indépendant de la croissance économique.

D'une part, certains auteurs avancent des arguments qui soutiennent un impact négatif de la croissance démographique sur la croissance économique. Thomas Malthus est probablement le premier économiste qui, il y a plus de 200 ans, à soulever l'idée selon laquelle la forte croissance démographique pèserait lourdement sur les disponibilités alimentaires, ce qui réduirait le niveau de vie des populations. Cette conception de l'effet négatif de la croissance démographique sur le bienêtre économique est appelée le "piège malthusien ». L'effet d'étranglement de la croissance démographique sur la croissance économique est soutenu empiriquement par plusieurs travaux dont ceux de Barro et Sala-i-Martin (2004). Avec des données portant sur 87 pays et couvrant la période de 1960 à 2000, ils ont montré qu'une augmentation des taux de fécondité de $1 \%$ entraine la baisse des taux de croissance de $0,006 \%$.

D'autre part, d'autres auteurs défendent l'idée selon laquelle la croissance démographique est indépendante de la croissance économique; cette dernière serait plutôt liée à d'autres facteurs dont la qualité des institutions politiques et économiques qui en est l'un des déterminants les plus importants (Norton, 2003). Cette thèse est soutenue également par les travaux de Easterly et Levine (2002) et Acemoglu et al. (200I).

Dans les années 1990, le débat sur l'effet de la croissance démographique sur la croissance économique est passé de la question de la croissance de la population en soi à la structure par âge de la population, i.e. la manière dont la population est répartie entre les différents groupes d'âge. En soutenant que les individus ont des comportements économiques qui varient à différents stades de la vie, Bloom et al. (200l) ont démontré que la structure par âge de la population d'un pays a un impact important sur ses performances économiques. Egalement à travers des analyses empiriques portant sur des données de l'Asie, Bloom et Williamson (1998) ont montré que la croissance démographique a un effet transitoire sur la croissance économique; cet effet s'opère seulement lorsque les populations dépendantes et en âge de travailler évoluent à des rythmes différents.
L'effet direct de la santé et des facteurs démographiques (notamment la fécondité) et économiques sur la croissance économique est étudié à travers plusieurs autres travaux. Utilisant un modèle de croissance, Bloom et al. (2010) ont analysé l'effet de la santé et des changements démographiques sur la croissance économique de la Chine et de l'Inde. Pour ces auteurs, les principaux facteurs qui sont à la base du décollage économique de ces deux pays sont l'amélioration de la santé, l'ouverture commerciale plus grande mais aussi l'augmentation du ratio main d'œuvre sur population. Plusieurs autres auteurs ont aussi trouvé une relation robuste entre le taux de fécondité et le développement économique (Fry et Mason (1982), Kelley et Schmidt (1996), Higgins (1998), Bloom et Finlay. (2009), entre autres): la baisse du taux de fécondité serait une opportunité de croissance économique car elle augmenterait la productivité et favoriserait l'épargne. Cette augmentation de la croissance découlant de la transition démographique est ce qu'on appelle le dividende démographique (Bloom et Williamson, 1998; Bloom et al., 200I, 2003; Mason, 200I).

Ainsi, le dividende démographique est l'accélération de la croissance économique d'un pays qui résulte de l'évolution de la structure par âge de sa population. Il est mesuré par la croissance du ratio de soutien qui représente la proportion de travailleurs par rapport aux consommateurs. En raison principalement du grand nombre d'enfants, le ratio de soutien est faible dans un pays à fort taux de fécondité. Et lorsque le taux de fécondité baisse rapidement, cela entraîne des changements dans la structure par âge de la population au sein de laquelle on observe désormais moins d'enfants et plus de personnes en âge de travailler. La charge familiale se réduit considérablement dans un premier temps. Mais elle va augmenter dans un deuxième temps en raison, cette fois-ci, de l'allongement de la durée de vie de la population. II existe donc entre ces deux phases une période intermédiaire où le ratio de soutien est élevé. La hausse du ratio de soutien signifie que la proportion de travailleurs par rapport aux consommateurs augmente et que, toutes choses égales par ailleurs, le revenu par consommateur augmente également (Lee et Mason, 2007). Cette période intermédiaire est donc une réelle opportunité de croissance économique et de développement humain plus rapide pour le pays, du fait que plus de ressources sont disponibles pour être investies dans le développement économique et le bien-être des familles. 
Par ailleurs, des auteurs se sont également penchés sur les effets d'un changement de la structure par âge de la population sur la croissance économique dans les pays africains. Ils ont montré qu'une augmentation de la part des personnes en âge de travailler par rapport à la population dépendante sera bénéfique pour la croissance économique de ces pays (Bloom et al. 2007; Mason et al. 20 I0; Van Der Ven et Smits, 20II; Drummond et al. 2014). Plus précisément, ces pays peuvent potentiellement bénéficier d'une population plus productive dès lors que le rapport des producteurs effectifs sur les consommateurs effectifs augmente. En outre, selon Drummond et al. (2014), l'ampleur de l'impact du dividende démographique dans les pays africains peut être plus importante que celle observée dans les économies matures.

Cependant, l'obtention de ce dividende démographique n'est pas automatique; elle nécessite plusieurs conditions préalables. Le dividende démographique n'est pas seulement subordonné aux taux de mortalité et de fécondité, il exige également que des investissements soient faits dans des domaines clés de l'économie. Comme le montre l'expérience récente des pays d'Asie de l'Est, le plein effet du dividende démographique pour un développement économique requiert la mise en œuvre des politiques efficientes concernant notamment l'ouverture au commerce international, la flexibilité du marché de l'emploi, l'éducation, la santé publique.

En effet, selon selon Bloom et al. (2000) et Mason (200I), les pays d'Asie de l'Est ont su profiter du dividende démographique parce qu'ils ont investi massivement et longuement dans l'éducation et la planification familiale. Ils ont en plus mené les réformes et les initiatives économiques nécessaires et ont intégré avec succès les femmes dans le marché du travail. Ceci a eu des effets positifs sur la croissance des pays de cette région et leur a permis de réduire considérablement la pauvreté et de se hisser presque au rang des pays développés. Lee et Mason (2006) estiment à plus $40 \%$ la contribution des dividendes démographiques à la croissance effective du PIB réel durant le " miracle économique » des pays de l'Asie de l'Est entre 1970 et 2000.

Leur exemple montre qu'un certain nombre de facteurs sont essentiels: la promotion des exportations pour permettre l'emploi rémunéré d'une force de travail en croissance rapide; la stabilité macroéconomique et le développement d'institutions financières encourageant l'épargne pour favoriser l'investissement; l'augmentation des dépenses d'éducation, permise par les changements de la structure par âge de la population et reposant sur une forte priorité à l'éducation dans les politiques de développement ; l'élimination des discriminations envers les femmes, par des politiques législatives et institutionnelles adaptées, afin qu'elles puissent contribuer davantage à la vie économique et sociale (Mason, 200I). En somme, les changements démographiques ont créé des opportunités de développement que les pays d'Asie de l'Est ont su saisir grâce à la mise en œuvre de politiques sociales et économiques favorables à l'augmentation du capital humain et l'accroissement de l'épargne et de l'investissement. Cette efficience des politiques publiques nécessite, selon Bloom et al. (2003), une bonne gouvernance afin d'améliorer l'efficacité des politiques sectorielles, la promotion d'un Etat de droit et la limitation de la corruption.

\section{METHODOLOGIE}

\section{Cadre conceptuel et méthodologique}

L'équation empirique utilisée dans cette étude est celle de Bloom et Canning (2004), qui est une équation standard de convergence conditionnelle permettant de déduire une relation entre la croissance du revenu par tête et les variables démographiques. Elle est fondée sur des modèles de croissance du revenu par travailleur de type Barro et Sala-i-Martin (2004), et est formulée en équation estimable en liant la croissance du revenu par travailleur à la croissance du revenu par habitant et la croissance de la population en âge de travailler.

Barro et Sala-i-Martin (2004) ont déduit de la littérature économique et ont largement discuté l'équation de croissance

$$
g_{y}=\lambda\left(X \beta+w_{0}-y_{0}\right)+g_{w} \text { qui sert de base à }
$$

notre estimation. Le log du revenu par travailleur est noté $z$, et la croissance du revenu par travailleur est notée $g_{z}$. L'équation établit que, sur une période donnée, la croissance du revenu par travailleur est liée au gap entre le niveau à l'état d'équilibre du revenu par travailleur $z^{*}$ et le niveau du revenu par travailleur en début de période $z_{0}$. $\lambda$ est un paramètre indiquant la vitesse d'ajustement vers l'état d'équilibre. Elle peut être reformulée comme suit:

$$
g_{z}=\lambda\left(z^{*}-z_{0}\right)
$$

En retour, le revenu par travailleur à l'état d'équilibre est une fonction de plusieurs variables ayant potentiellement une influence sur la productivité du travail. Ces dernières comprennent des indicateurs de santé et d'éducation qui déterminent la qualité de la main d'œuvre, ou facteurs invariants dans le temps tels que le climat, la géographie, la culture. En désignant ces déterminants de la productivité du travail par le vecteur $X$ et le vecteur de paramètres associés aux variables par $\beta$ 
(i.e. $z^{*}=X \beta$ ), l'équation (I) peut être réécrite comme suit:

$$
g_{z}=\lambda\left(X \beta-z_{0}\right)
$$

Afin de relier le modèle de croissance du revenu par travailleur $g_{z}=\lambda\left(X \beta-z_{0}\right)$ à la croissance du revenu par tête et aux variables démographiques, on utilise l'identité simple suivante:

$$
\frac{Y}{N}=\frac{Y}{L} \times \frac{L}{N}
$$

Où $Y$ est le revenu, $N$ la population totale et $L$ la population en âge de travailler.

Le terme $\frac{L}{N}$ est donc la proportion de la population en âge de travailler. Il désigne également

\section{le ratio de soutien démographique.}

Ainsi, cette identité établit que le revenu par tête est égal à la productivité du travail fois le taux de soutien démographique.

En appliquant la transformation logarithmique à l'équation (3) on obtient:

$$
\log \left(\frac{Y}{N}\right)=\log \left(\frac{Y}{L}\right)+\log \left(\frac{L}{N}\right)
$$

Puis en posant $y=\log \left(\frac{Y}{N}\right), \quad z=\log \left(\frac{Y}{L}\right)$ et $w=\log \left(\frac{L}{N}\right)$, il s'ensuit que

$$
z=y-w
$$

De plus, en termes de croissance, l'équation (4) donne:

$$
g_{y}=g_{z}+g_{w}
$$

avec $g_{y}$ la croissance du revenu par tête, $g_{z}$ la croissance de la productivité et $g_{w}$ la croissance du ratio de soutien démographique.

En remplaçant (5) et (6) dans (2), on obtient:

$$
g_{y}=\lambda\left(X \beta+w_{0}-y_{0}\right)+g_{w}
$$

Notre modèle empirique est basé sur cette dernière équation. Celle-ci stipule que, au cours du temps, le niveau initial du ratio de dépendance démographique et le taux de croissance du ratio de soutien démographique devraient être positivement liés à la croissance du revenu par tête. Ceci vient s'ajouter à l'impact des autres variables pouvant influencer la productivité du travail. II est à noter également que le vecteur $X$ peut contenir des facteurs invariants dans le temps. Mais comme notre analyse porte sur un seul pays, tous ces facteurs invariants dans le temps sont éliminés de l'équation, ainsi les facteurs à l'état initial notamment $w_{0}$ et $y_{0}$.
Finalement, notre modèle empirique est spécifié comme suit:

$$
g_{y}=\lambda X \beta+g_{w}+\varepsilon
$$

où $X$ est un vecteur de variables susceptibles d'affecter la productivité du travail. II inclut le capital humain et le capital physique (qui sont approximés par le taux de scolarisation et la consommation d'électricité respectivement) et autres variables de contrôle.

L'hypothèse du cycle de vie postule que les travailleurs ont une épargne positive tandis que les jeunes et les vieux consomment plus que ce qu'ils gagnent. Ainsi une hausse du ratio de soutien démographique est susceptible d'être associé à une augmentation des épargnes et donc du stock de capital.

Toutefois, le ratio de soutien démographique, en tant qu'une approximation du rapport entre les travailleurs et les consommateurs nets, souffre d'importantes lacunes qui sont essentiellement de deux ordres. La première insuffisance concerne le terme $L$ au numérateur qui sert de proxy aux travailleurs. Le ratio de soutien démographique considère que toute personne en âge de travailler est un actif occupé. De plus, il suggère que les enfants de moins de 15 ans ainsi que les personnes âgées de 65 ans ou plus sont économiquement dépendants. De ce fait, le ratio ne peut être influencé que par un seul facteur: la modification de la limite théorique séparant l'âge de travailler et l'âge de la retraite.

Mais en réalité dans toute société, on observe que toutes les personnes en âge de travailler ne sont pas forcément actives, encore moins actives occupées. En effet, la société compte au sein de sa population en âge de travailler des chômeurs, des invalides ou encore des personnes au foyer. On assiste aussi de plus en plus à une augmentation de la période de formation pour une vie productive. De ce fait les adolescents et les jeunes adultes restent plus longtemps à l'école et demeurent donc hors de la population active plus longtemps, poussant ainsi la limite du début la vie active au-delà de 15 ans. Par conséquent, il est invraisemblable que toutes les personnes âgées de 15 à 64 ans soient économiquement actives. De plus, s'agissant des personnes du troisième âge, dans de nombreuses populations, tout le monde n'arrête pas de travailler à 65 ans. En outre, certaines personnes âgées disposent de leurs propres ressources économiques et fournissent un soutien à leurs enfants adultes, et ne sont donc pas économiquement dépendantes.

La seconde insuffisance concerne le terme du dénominateur $N$ qui désigne la population totale. En prenant la population totale en terme absolu pour approximer le nombre de consommateurs, le ratio de soutien attribue à toutes les personnes, quels que 
soient leurs âges, un niveau de consommation égal. Ainsi, un enfant de moins de 5 ans aurait les mêmes besoins de consommation qu'un adulte de 40 ans par exemple. Or il est démontré que la consommation augmente considérablement de l'enfance jusqu'au début de l'âge adulte, stade auquel elle atteint son niveau maximal (Nations Unies, 2007). Les besoins et les niveaux de consommation diffèrent donc suivant l'âge des individus. L'indicateur du nombre de consommateurs est donc biaisé lorsqu'on essaie de l'approcher avec la population totale en terme absolu.

Pour pallier ces lacunes, plusieurs auteurs dont Cutler et al. (1990) notamment, ont proposé une alternative de calcul du ratio de soutien qui tient compte de la population active effectivement occupée (ou "producteurs effectifs") et du nombre des " consommateurs effectifs".

Désignons par $L^{\prime}$ le nombre de " producteurs effectifs " et par $N^{\prime}$ celui de "consommateurs effectifs ». Pour obtenir $\boldsymbol{L}^{\prime}$, la population en âge de travailler est pondérée par (a) le revenu moyen par âge et (b) les estimations des taux de participation au marché du travail selon l'âge. Le nombre de " consommateurs effectifs" $N^{\prime}$ se calcule, quant à lui, en pondérant le nombre de consommateurs (i.e. la population totale) par la variation des besoins de consommation en fonction de l'âge.

Ce nouvel indicateur (le rapport $\frac{\boldsymbol{L}^{\prime}}{\boldsymbol{N}^{\prime}}$ ) est appelé ratio de soutien économique et s'obtient par la formule suivante: $\frac{L^{\prime}}{N^{\prime}}=\frac{\sum \gamma(a) P(a, t)}{\sum \varphi(a) P(a, t)}$ avec $\gamma$ la productivité moyenne par âge et $\boldsymbol{\varphi}$ la distribution des besoins de consommation en fonction de l'âge.

Contrairement au taux de dépendance démographique, le ratio de soutien économique ne traduit pas seulement le rapport mathématique entre les différents groupes d'âge, mais prend en compte la situation économique (participation à l'activité économique) et les besoins des individus. Et cela constitue une différence énorme tant au niveau du nombre de travailleurs qu'au niveau du nombre de consommateurs. Si l'on compare avec le taux de dépenandance démographique qui ne peut être influencé que par une modification des limites d'âge, ou les effets de la migration, le nombre de variables ayant un impact sur l'évolution du ratio de soutien économique s'avère beaucoup plus élevé: (I) le chômage, (2) pouvoir d'achat des travailleurs (3) dynamisme du marché du travail, (4) participation des femmes au marché du travail, (5) les travailleurs de la diapora, etc.
Le ratio de soutien économique a donc le privilège de refléter à la fois l'effet de la structure d'âge et les profils par âge de consommation et de revenu. II donne une description plus riche et plus précise de la réalité. Pour ces raisons, le ratio de soutien économique sera considéré à la place du taux de dépendance démographique dans la suite du document, sauf indication contraire.

\section{Les données}

Pour analyser l'impact du dividende démographique sur la croissance économique du Sénégal, nous considérons un modèle économétrique qui reprend l'équation (8) où $g_{y}$ étant la croissance du PIB par tête, $g_{w}$ celle du ratio de soutien économique, et $X$ un vecteur de variables de contrôle formé par d'autres déterminants de la croissance à savoir le taux d'investissement, le taux d'inflation, le capital humain, le capital physique. Le taux de scolarisation et la consommation d'électricité servent de proxy au capital humain et au capital physique respectivement.

Les données utilisées s'étalent entre 1980 et 2010. Elles proviennent toutes de World Development Indicators (WDI) de la Banque mondiale, à l'exception du ratio de soutien économique qui est construit par le Centre de Recherche en Economie et Finance Appliquées de Thiès (CREFAT) à partir des estimation NTA (National Transfer Accounts).

\section{RESULTATS EMPIRIQUES}

Du fait que les données sont en séries temporelles, nous allons effectuer des tests de stationnarité et éventuellement de cointégration sur ces dernières. En fonction des résultats de ces tests et selon que les séries sont stationnaires ou cointégrées, l'estimation des moindres carrés ordinaires ou celle d'un modèle à correction d'erreur sera appliquée. Les soussections suivantes présentent successivement les résultats des tests de racine unitaire et de cointégration, et de l'estimation de l'équation empirique retenue dans la section précédente. Mais avant cela, nous présentons une comparaison entre l'évolution du ratio de soutien démographique et celle du ratio de soutien économique.

Evolution comparative des ratios de soutien démographique et économique

En tant que rapport mathématique entre différents groupes d'âge, il est évident que le ratio de soutien démographique ne présente pas forcément la même allure que le ratio de soutien économique qui prend en compte le profil par âge de la consommation et du revenu. C'est ce que révèle le graphique ci-dessous qui présente les évolutions des deux ratios sur la période 1980 à 2010. 


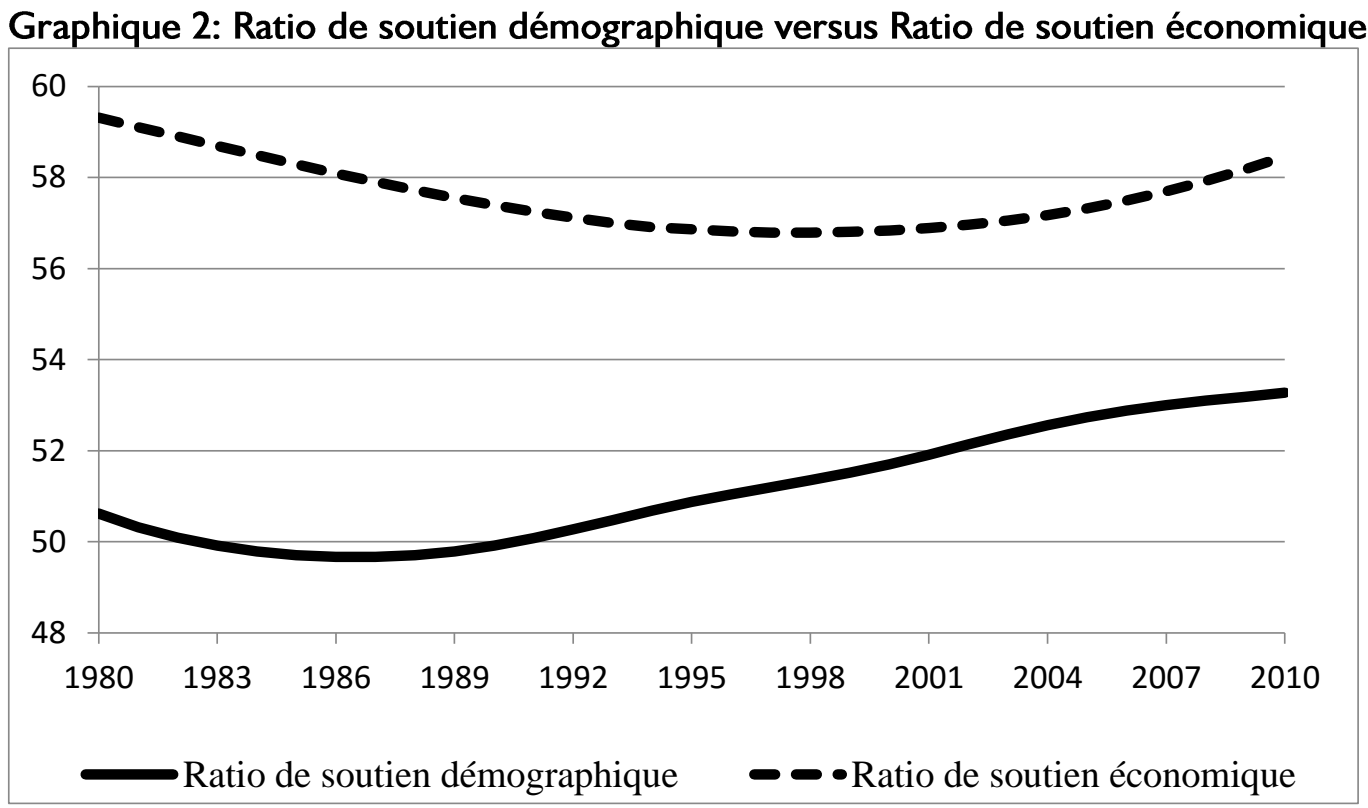

Source: WDI 2015, ESPS I, Calculs des auteur.

Le graphique montre que les deux ratios ont une tendance baissière depuis le début de la période, mais la baisse du ratio de soutien démographique s'est arrêté vers 1988, alors que celle du ratio de soutien économique s'est poursuivie jusqu'au début des années 2000.

A partir de 1989, on observe une évolution croissante du ratio de soutien démographique. C'est donc certainement à partir de ce moment que les effets de la baisse de la fécondité commencent à se sentir sur la structure par âge de la population sénégalaise: la proportion des personnes en âge de travailler augmente légèrement plus vite. Toutefois, cet accroissement de la population active n'est pas accompagné par une augmentation la proportion des travailleurs effectifs, c'est pour cette raison que le ratio de soutien économique continue à décroitre. C'est donc une situation difficile que les travailleurs ont enduré à cette époque car ils doivent prendre en charge de plus en plus de personnes. II faut attendre le début des années 2000 pour observe une amélioration du ratio de soutien économique qui va désormais prendre une tendance ascendante.

Tests de racine unitaire et de cointégration sur les variables

\section{$\checkmark \quad$ Tests de racine unitaire}

Le tableau 2 présente les résultats du test de Dickey Fuller Augmenté. II montre que les variables Ratio de soutien économique et Taux d'inflation sont stationnaires. Pour toutes les autres variables, l'hypothèse selon laquelle les séries présentent une racine unitaire est acceptée. Toutefois leurs différences premières sont stationnaires: elles sont donc intégrées d'ordre I. 
Tableau 2: Résultats des tests de racine unitaire

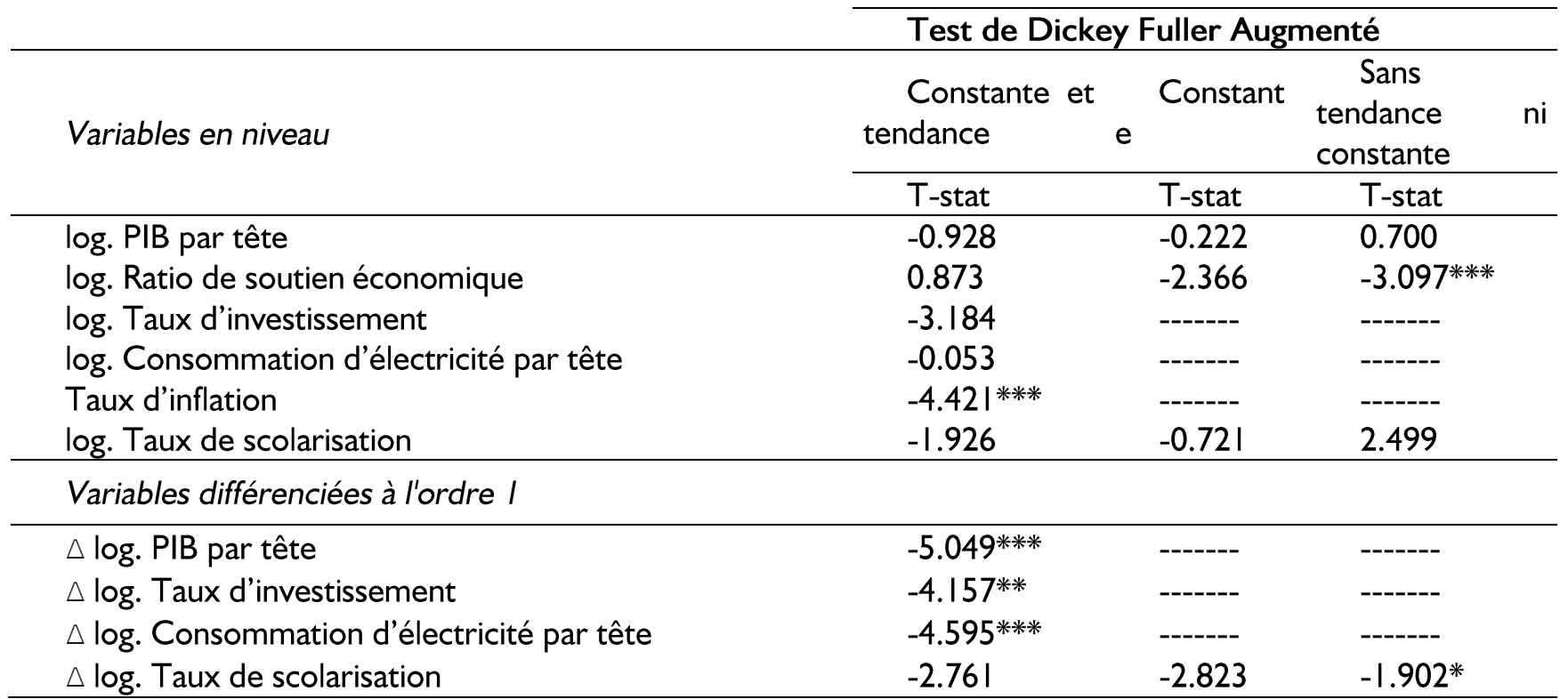

***, ** et * indiquent le rejet de l'hypothèse nulle de présence de racine unitaire à $1 \%, 5 \%$ et $10 \%$ respectivement.

Source: Calculs des auteurs

Test de cointégration

Les résultats de l'analyse de la co-intégration entre les variables du modèle sont présentés dans le tableau 3. La co-intégration est analysée en utilisant le test de Engle-Granger.

Tableau 3: Résultats du test de cointégration

\begin{tabular}{|c|c|c|c|c|c|}
\hline \multicolumn{3}{|c|}{ Engle-Granger test for cointegration } & \multicolumn{2}{|c|}{$\begin{array}{ll}N(\text { lst step }) & = \\
N(\text { test }) & =\end{array}$} & $\begin{array}{l}31 \\
30\end{array}$ \\
\hline & Test & $1 \frac{\circ}{8}$ Critical & $5 \%$ Critical & \multicolumn{2}{|c|}{10 \% Critical } \\
\hline & Statistic & Value & Value & \multicolumn{2}{|r|}{ Value } \\
\hline $2(t)$ & -6.326 & -6.569 & -5.661 & \multicolumn{2}{|r|}{-5.226} \\
\hline
\end{tabular}

Source : Calculs de l'auteur

Le tableau 3 ci-dessus indique l'existence de relation de cointégration à un seuil de $5 \%$. II existe alors, sur la base de ces résultats une relation de long terme entre les variables PIB par tête, le ratio de soutien économique, la consommation d'électricité, le taux d'investissement, le taux d'inflation et le taux de scolarisation au Sénégal sur la période de l'étude.

Estimations des équations de long et de court terme

L'approche du modèle à correction d'erreur est adoptée pour estimer l'impact du dividende démographique sur la croissance économique au Sénégal. Cette approche se justifie par l'existence de relation de cointégration entre les variables. Empiriquement, l'effet du dividende démographique est mesuré par la part de la population en âge de travailler ou par le ratio de soutien économique. La variation du PIB réel par tête est régressée sur le ratio de soutien économique et un ensemble de variables de contrôle standard (démographiques et économiques) pour étudier l'impact du dividende démographique sur la croissance économique du Sénégal. Le tableau 4 présente les résultats de l'estimation de l'équation de long terme et de court terme. 
Tableau 4: Résultats de l'estimation de l'équation de long et de court terme

\begin{tabular}{|c|c|c|}
\hline log. PIB par tête & $\begin{array}{l}\text { Equation de } \\
\text { Long terme }\end{array}$ & Court terme \\
\hline log. Ratio de soutien économique & $\begin{array}{l}3.693 * * * \\
(0.691)\end{array}$ & $\begin{array}{l}-0.0678 \\
(0.318)\end{array}$ \\
\hline log. Taux d'investissement & $\begin{array}{l}0.00115 \\
(0.0363)\end{array}$ & $\begin{array}{l}0.0280 \\
(0.0356)\end{array}$ \\
\hline log. Consommation d'électricité par tête & $\begin{array}{l}-0.0927 \\
(0.0604)\end{array}$ & $\begin{array}{l}-0.0570 \\
(0.0432)\end{array}$ \\
\hline Taux d'inflation & $\begin{array}{l}-0.000373 \\
(0.000659)\end{array}$ & $\begin{array}{l}-0.000529 \\
(0.000559)\end{array}$ \\
\hline log. Taux de scolarisation & $\begin{array}{l}0.293 * * * \\
(0.0751)\end{array}$ & $\begin{array}{l}0.0632 \\
(0.175)\end{array}$ \\
\hline $\operatorname{ECM}(-I)$ & & $\begin{array}{l}-1.205^{* * * *} \\
(0.269)\end{array}$ \\
\hline Constante & $\begin{array}{l}4.876 * * * \\
(1.4 \mid 3)\end{array}$ & $\begin{array}{l}-0.0335 \\
(0.176)\end{array}$ \\
\hline Observations & 31 & 30 \\
\hline $\mathrm{R}^{2}$ ajusté & 0.9182 & 0.4799 \\
\hline F-Stat & 57.16 & 4.82 \\
\hline Prob > F & 0.0000 & 0.0021 \\
\hline
\end{tabular}

Standard errors in parentheses $* * * p<0.01,{ }^{* *} p<0.05,{ }^{*} p<0.1$

Source: Nos Calculs

Le coefficient de correction d'erreur ECM (-I) représente la vitesse de l'ajustement de la relation de court terme vers l'équilibre de long terme. Le signe négatif et la significativité de ce coefficient à $1 \%$ $(E C M=-I, 205)$ traduit l'existence d'un mécanisme à correction d'erreur. Tous les déséquilibres entre le taux de croissance économique par tête et les variables explicatives se compensent à long terme, de sorte que les séries retrouvent des évolutions similaires. Ainsi, les déséquilibres entre les niveaux désirés et effectifs du taux de croissance par tête du PIB sont entièrement résorbés l'année qui suit le choc par un effet de feed-back. Ceci valide donc la spécification du modèle à correction d'erreur.

Concernant la relation de long terme, la significativité du coefficient de la variable Ratio de soutien économique montre l'existence d'un lien empirique à long terme entre le dividende démographique et la croissance économique au Sénégal. Les résultats indiquent que le dividende démographique influence positivement la croissance économique à long terme. En effet, une augmentation du rapport des travailleurs effectifs sur les consommateurs effectifs de $1 \%$ se traduit par un accroissement du PIB par tête de 3,7\% à long terme.

Outre le ratio de soutien, l'on note que le capital humain est un facteur important de croissance économique. En effet, la variable Taux de scolarisation, proxy du capital humain, a un impact positif et significatif sur le PIB par tête. Toute augmentation du taux de scolarisation de $1 \%$ est associée à une hausse du taux de croissance par tête de $0,4 \%$ à long terme. Ce résultat tend ainsi à valider l'effet positif de l'accumulation du capital humain sur la croissance économique à long terme. En effet, l'accumulation du capital humain permet de soutenir la croissance à long terme en agissant directement sur la productivité de la main-d'œuvre (Sen, 2000).

\section{Discussions}

Comme la plupart des pays africains, le Sénégal est entré dans la deuxième phase de la transition démographique avec un délai et une vitesse plus rapides par rapport aux pays d'Europe (où le processus a duré un siècle et demi environ). La transition démographique apporte avec elle des 
changements dans la structure de la population. En effet, ayant démarré vers la fin des années 1970, la baisse des taux de mortalité est en train d'être suivie actuellement par le déclin des taux de fécondité. Cela crée une grande cohorte de jeunes et de personnes en âge de travailler dont la proportion va devenir de plus en plus importante au fil des années. Cet accroissement de la proportion de personnes en âge de travailler se traduit par une hausse du ratio de soutien démographique.

Cette modification de la structure de la population au cours de la transition démographique a des conséquences sur la croissance économique. En faisant l'hypothèse que toute personne ayant atteint l'âge de travailler est employée de façon productive, l'accroissement de la population en âge de travailler entraine une hausse du ratio de soutien économique, ce qui contribue à une augmentation de la production par tête et de l'épargne. Cependant, l'hypothèse selon laquelle toute personne en âge de travailler est systématiquement employée de façon productive, est très souvent violée dans la réalité. Pour cette raison, dans le cadre de ce travail, nous avons calculé le ratio de soutien économique à partir du rapport des " travailleurs effectifs" sur les " consommateurs effectifs "; les " travailleurs effectifs" étant obtenus par pondération de la population en âge de travailler par les taux de participation au marché du travail selon l'âge, et les "consommateurs effectifs ", par pondération de la population totale par les variations des besoins de consommation en fonction de l'âge.

Empiriquement, l'effet du dividende démographique est capté à travers le ratio de soutien économique. Les estimations économétriques effectuées révèlent que l'accroissement de ce ratio a un impact positif et significatif sur la croissance du PIB réel par tête à long terme. Et cet impact est de l'ordre de $3 \%$ suite à une variation de $1 \%$ du ratio de soutien économique. Autrement dit, le dividende démographique agit positivement sur la croissance économique du Sénégal. Ceci montre que la transition démographique représente une opportunité majeure pour l'économie sénégalaise puisqu'il y a proportionnellement moins d'inactifs effectifs à charge, et les possibilités de création de valeur par une force de travail abondante sont plus importantes. De plus, au-delà de l'effet direct de cette modification de la structure par âge sur l'offre de travail, un effet indirect peut également se produire à travers un changement de la participation des femmes au marché du travail. En effet, avec la baisse de la fécondité (qui est un facteur déterminant de la transition démographique), la nécessité d'élever des enfants devient moindre et le taux de dépendance des plus jeunes devient également faible.
En conséquence, plus de femmes peuvent entrer sur le marché du travail lorsqu'elles disposent de compétences requises.

Au Sénégal, les résultats montrent un impact positif de la variation de la part des travailleurs effectifs sur le PIB par tête. Ceci peut être expliqué par plusieurs raisons parmi lesquelles deux sont évoquées ici. Premièrement, une part des travailleurs élevée qui entraîne une hausse du ratio de soutien économique et donc une baisse du ratio de dépendance démographique ${ }^{5}$ (nombre de personnes jeunes et âgées par rapport au nombre de personnes en âge de travailler), devrait impliquer un niveau d'épargne par travailleur plus élevé, et une productivité plus grande. En revanche, lorsque la proportion de jeunes ou de personnes âgées en situation de dépendance est élevée, le pays tend à consacrer une part relativement importante de ses ressources à ces catégories, et en limitant très souvent la croissance économique. Bloom et al. (2003) ont montré que le taux d'épargne est faible dans les pays à fort taux de dépendance démographique, ce qui conduit inévitablement à une baisse de la productivité lorsque la formation de capital domestique est contrainte par l'épargne.

Deuxièmement, une part des travailleurs élevée contribue à une augmentation du revenu du travail au plan national, ce qui conduit directement à un niveau élevé du revenu par tête. Ainsi, lorsque la partie de la population qui travaille et épargne est relativement importante, le Sénégal peut bénéficier d'une accélération de la croissance des revenus découlant d'une population plus importante, de l'accumulation accélérée du capital et de dépenses réduites pour la population dépendante.

Ce résultat est corroboré par les travaux de Bloom et al. (2000) effectués sur l'Asie de l'Est qui représente l'expérience la plus accomplie du dividende démographique. Ce dernier a un impact de l'ordre de $25 \%$ sur la croissance du revenu réel par tête durant la période 1965 - 1990, période où la population active augmenta 4 fois plus vite que les populations dépendantes.

Par ailleurs, le Sénégal présente aujourd'hui les mêmes profils démographiques qui prévalaient 50 ans auparavant dans les pays d'Asie de l'Est. Par exemple, l'actuel taux d'accroissement naturel de population sénégalaise ( $2,5 \%$ par année) est semblable, voire inférieur à celui de la Corée du Sud (2,7\% par an), du Singapour (2,7\% par an) ou encore de la Thaillande

\footnotetext{
${ }^{5}$ |l existe un lien négatif entre le ratio de soutien et le ratio de dépendance démographique : l'augmentation de la part des travailleurs effectifs équivaut à une baisse du ratio de dépendance.
} 
(3,1\% par an) au cours de la période 1960-19656. Mais grâce à des programmes de panification familiale qui sont décisifs, la Corée du Sud, le Singapour et la Thaillande ont réussi, de façon spectaculaire et en un temps record, à maitriser la croissance de leur population qui est estimée à $1 \%, 1,3 \%$ et $1,1 \%$ respectivement en 1990; puis à $0,5 \%, 0,5 \%$ et $0,7 \%$ respectivement en 2000.

L'indice synthétique de fécondité de l'Asie de l'Est qui est 5,I enfants par femme en 1960-1965 est comparable à celui du Sénégal de nos jours (5 enfants par femme en 20II). Les enquêtes démographiques ont montré pour l'Asie de l'Est, que l'indice synthétique de fécondité a chuté à I,9 enfant par femme en 1990 (soit une baisse de 3,2 de l'ISF en 25 ans), puis à I,7 enfant en 2000.

Aussi, la situation actuelle du Sénégal en matière de mortalité est-elle similaire à celle observée en Asie orientale 40 ans plus tôt. En effet, la mortalité infantile dans cette région de l'Asie est $56 \%$ o au début des années 1970, alors qu'elle est estimée à $47 \%$ ou Sénégal en 2011 .

En définitive, d'après ce qui précède, le Sénégal dispose des mêmes principes démographiques qui avaient précédé le " miracle économique " des pays de l'Asie de l'Est. De plus, des travaux de recherche révèlent que la fenêtre d'opportunités liées au dividende démographique est en train de s'ouvrir pour le Sénégal (Dramani et Ndiaye, 2012). C'est donc le meilleur moment de mettre en place des réformes appropriées et un environnement politique et institutionnel adéquat pour créer et tirer profit du dividende démographique. Les paragraphes qui suivent font des recommandations de politiques qui sont indispensables dans ce sens.

\section{Conclusion et implications de politiques}

L'objectif visé à travers ce travail est d'examiner si le dividende démographique influence positivement la croissance économique du Sénégal, et de déterminer l'ampleur de cet impact dans le cas échéant. Pour ce faire, l'indicateur du ratio de soutien économique est conçu pour capter l'effet du dividende démographique, i.e. du changement de la structure par âge de la population sénégalaise tout en tenant compte du profil de consommateur et de revenu du travail selon les âges.

A partir d'un modèle à correction d'erreur sur des données couvrant la période 1980 à 2010, l'on s'aperçoit de l'existence d'un lien entre le dividende

6 Dans cette section, toutes les statistiques démographiques concernant les pays d'Asie de l'Est sont obtenues à partir de l'article publié en 2009 par Attané et Barbieri: "La démographie de l'Asie de l'Est et du Sud-Est des années 1950 aux années 2000: Synthèse des changements et bilan statistique ».

http://aps.journal.ac.za démographique et le développement économique au Sénégal. Plus précisément, la variation du ratio de soutien économique a un impact positif et significatif sur la croissance économique du Sénégal; toute augmentation du ratio d'un point de pourcentage est associée à une hausse du revenu par tête de 3,7\%. Le dividende démographique s'avère donc être une source de croissance pour l'économie sénégalaise.

La transition démographique dont le point de départ est la baisse des taux de mortalité, puis de fécondité, permet d'accroitre le ratio de soutien. Etant donné qu'il est au début de ce processus de transition, le Sénégal pourrait mieux capitaliser son dividende démographique en mettant en œuvre des politiques pour accompagner la baisse de la fécondité. II s'agit notamment des investissements dans les secteurs de l'éducation, la santé, la planification familiale, l'emploi et la bonne gouvernance qui sont des points critiques pour tirer pleinement profit du dividende démographique. Ainsi, la mise en œuvre des mesures ci-dessous seront bénéfiques pour la nation.

Planification familiale: La transition démographique constitue la première étape vers l'atteinte du dividende démographique. Le passage d'une mortalité et d'une natalité élevées à des taux faibles est ainsi indispensable. Bien que le Sénégal ait fait des progrès significatifs en matière de réduction de la mortalité et de la natalité, il continue à maintenir des taux de fécondité relativement élevés rendant ainsi la transition démographique très lente, ce qui peut hypothéquer l'atteinte du dividende démographique. Tant que la fécondité et le taux d'accroissement naturel qui en découle demeurent élevés, la taille de la population en âge scolarisable sera plus importante que la population en âge de travailler. L'Etat, aussi bien les familles, aura du mal à investir dans la santé et l'éducation des enfants et aura peu de ressources supplémentaires pour investir dans les infrastructures nécessaires pour stimuler la création d'emplois et la croissance économique. II serait donc très utile de renforcer les services en matière de planning familial pouvant aider les familles à espacer mieux les naissances notamment.

Santé: Bien que le planning familial soit nécessaire pour établir les conditions du dividende démographique, l'Etat doit également investir dans la santé publique. L'amélioration de la santé surtout de la survie de l'enfant peut entrainer une augmentation de la demande de planning familial. Plusieurs travaux de recherche, notamment ceux de Joshi (20I2), montrent qu'en Afrique subsaharienne, les familles pourraient avoir moins d'enfants lorsqu'elles auront la garantie que chacun d'entre eux jouisse d'une meilleure chance de survie. II est donc indispensable 
de renforcer les systèmes de santé dans leur ensemble. L'amélioration de l'hygiène, des programmes de vaccination et l'usage d'antibiotiques permettent non seulement de réduire la mortalité, mais aussi de maintenir la population en bonne santé et d'être potentielle productive.

Education: II est capital de transformer la population jeune en force de travail productive. L'accès à l'éducation ainsi que la qualité de celle-ci doivent être améliorés. Les systèmes éducatifs doivent garantir que les jeunes terminent leur scolarité et qu'ils acquièrent les compétences nécessaires pour s'adapter au marché du travail en évolution.

L'égalité des sexes dans l'éducation est également à promouvoir. Pour cela, il faut lutter contre le mariage précoce et sensibiliser les parents encore réticents à envoyer leurs filles à l'école. En effet, il s'est révélé que l'éducation des filles favorise l'amélioration de l'état de santé et la baisse de la fécondité. Les femmes non scolarisées qui se marient précocement, ont beaucoup d'enfants et utilisent peu ou mal les systèmes de soins. Inversement, l'effort de scolarisation des filles favorise un recul de l'âge au mariage, une baisse de la fécondité et de la mortalité des enfants. L'éducation des filles accroît en effet l'information en matière de santé, facilite l'adoption de comportements sanitaires favorables à une bonne santé de leurs enfants.

Il est également nécessaire que l'enseignement secondaire, l'enseignement supérieur et la formation professionnelle soient tous élargis et proposent des programmes pertinents afin que les jeunes acquièrent les compétences requises pour trouver un emploi productif.

Emploi et politiques économiques : Une main d'œuvre plus nombreuse et mieux qualifiée ne sert que si les travailleurs peuvent trouver des emplois. Ainsi, l'Etat doit mettre en place des politiques pour stimuler la création d'emplois; le marché du travail doit être modernisé avec une souplesse accrue en matière d'embauche, de mobilité professionnelle. II est aussi nécessaire de mettre en œuvre des politiques commerciales qui créent à la fois des marchés pour les produits locaux et réduisent les obstacles à l'importation de produits étrangers.

L'Etat doit également créer des opportunités économiques dans les milieux ruraux et pour les segments les plus pauvres de la population. Les programmes de microfinance dans les zones rurales peuvent apporter une amélioration des conditions de vie des ménages les plus pauvres. En effet, le fait d'accorder à ces ménages des prêts à faibles taux d'intérêt permet la création d'activités génératrices de revenu pour ces personnes qui sont en dehors des zones urbaines et de stimuler ainsi la croissance économique.

En somme, la transition démographique en cours représente une opportunité pour le Sénégal de réaliser le dividende démographique. En parvenant à planifier et à effectuer les investissements nécessaires dans les jeunes, le pays peut créer un cercle vertueux consistant à améliorer l'éducation, le capital humain et la productivité économique, ce qui conduit ainsi à l'atteinte du dividende démographique. A l'inverse, sans la mise en place effective de bonnes politiques ou si la transition est mal gérée, le pays risque de rater des occasions de croissance économique ou pire, il peut avoir à faire face à un chômage massif et à d'autres crises sociales en particulier lorsque l'économie ne crée pas d'emplois sur une échelle suffisante pour absorber ceux qui rejoignent le marché du travail.

\section{Références}

Acemoglu D., S. Johnson and J. Robinson (200I). "The colonial origins of comparative development: an empirical investigation ". American Economic Review 91:1369-140 I

Adjamagbo A. et P. Antoine (2002). "Le Sénégal face au défi démographique "Document de recherche DIAL/ CREFA.

ANSD (2015). " Rapport Projection de la population du Sénégal, 2013-2063 ». Bureau Etat Civil et Projections Démographiques - DRSD/DSDS.

ANSD et ICF International (20I2). Enquête Démographique et de Santé à Indicateurs Multiples au Sénégal (EDS-MICS) 2010-20II. Calverton, Maryland, USA; ANSD et ICF International.

Attané I. et M. Barbieri (2009). " La démographie de l'Asie de l'Est et du Sud-Est des années 1950 aux années 2000: Synthèse des changements et bilan statistique », Population, 64(I), p. 7154.

Banque Mondiale (20|4). Situation économique du Sénégal - Apprendre du passé pour avenir meilleur. Première édition

Barro R and X. Sala-I-Martin. (2004). Economic Growth. 2nd edition. Cambridge: MIT Press.

Bloom D. and D. Canning (2004) "Global Demographic Change: Dimensions and Economic Significance", NBER Working Paper 10817, NBER.

Bloom D., D. Canning, G. Fink, and J. Finlay. (2007). Realizing the Demographic Dividend: Is Africa any different? Program on the Global Demography of Aging - Harvard University.

Bloom D., D. Canning, L. Hu, Y. Liu, A. Mahal and W. Yip (20I0). " The contribution of Population Health and Demographic Change to Economic 
Growth in China and India ", Journal of Comparative Economics, 38: 17-33.

Bloom D, D. Canning, and P. Malaney (2000). "Demographic Change and Economic Growth in Asia " Population and Development Review, Vol. 26.

Bloom D., D. Canning and J. Sevilla (200I). "Economic growth and the demographic transition ». Working Paper 8685.

Cambridge, MA: National Bureau of Economic Research.

Bloom D., D. Canning and J. Sevilla (2003). The Demographic Dividend: A New Perspective on the Economic Consequences of Population Change. Santa Monica, California: RAND. MR1274

Bloom D. and J. Finlay (2009). "Demographic Change and Economic Growth in Asia ", Asian Economic Policy Review 4:45-64.

Bloom D. and J. Williamson (1998). "Demographic Transition and Economic Miracles in Emerging Asia ». World Bank Economic Review, I2(3).

Cutler D., J. Poterba, L. Sheiner and L. Summers (1990). " An Aging Society: Opportunity or Challenge? " Brooking Papers on Economic Activity 1990(I): I-73.

Diagne A. et G. Daffé (2002). Le Sénégal en quête d'une croissance durable. CREA-Karthala.

DPS et DHS - Macro International (1997). Enquête démographique et de santé au Sénégal 1997 (EDS III). Dakar: Calverton

Dramani L. and F. Ndiaye (20I2). "Estimating the First Demographic Dividend in Senegal: The National Transfers Account Approach », British Journal of Economics, Management and Trade 2(2): 39-59.

Drummond P., V. Thakoor, and S. Yu (20/4). "Africa Rising: Harnessing the Demographic

Dividend ", IMF Working Paper, WP//4// 43.

Easterly W. and R. Levine. (2002). " Tropics, germs, and endowments ». NBER Working Paper No. 9106. Carnegie-Rochester Conference Series on Public Policy.

Fry M. and A. Mason (1982). " The Variable Rate of Growth Effect in the Life-Cycle Model ", Economic Enquiry, Vol. 20.

Joshi S. (20I2), Reproductive Health and Economic Development: What Connections Should We Focus On? (Washington, DC: Population Reference Bureau)

Higgins M. (1998). « Demography, National Savings, and International Capital Flows », International Economic Review, Vol. 39.
Kelley A. and R. Schmidt (1996). "Saving, Dependency, and Development ", Journal of Population Economics, Vol. 9.

Lee R. and A. Mason (2006). "Les dividendes de l'évolution démographique " Finance et Développement, FMI, Septembre 2006.

Lee R. and A. Mason (2007). "Population ageing, wealth, and economic growth: demographic dividends and public policy ", Background Paper for the World Economic and Social Survey, Department of Economic and Social Affairs, United Nations

Mason A. (200I). "Population Change and Economic Development in East Asia: Challenges Met, Opportunities Seized ». California: Stanford University Press.

Mason A., O. Olaniyan and A. Soyibo (2010). Population and Economic Progress in Nigeria. Background Research report submitted to Harvard School of Public Health on the Nigerian Generation Next project

Nations Unies (2007). "Suivi de la situation démographique mondiale centré sur l'évolution des pyramides des âges et sur ses implications sur le développement ", Rapport du Secrétaire général, 07-2/ 286.

Ndiaye S. et M. Ayad. (2006). Enquête Démographique et de Santé au Sénégal 2005. Calverton, Maryland, USA; Centre de Recherche pour le Développement Humain [Sénégal] et ORC Macro.

Norton S. (2003). Population growth, economic freedom and the rule of law Stanford. CA: Hoover Press.

Pison G., K. Hill K. B. Cohen, et K. Foote (1997). "Les changements démographiques au Sénégal ". INED, collection Travaux et documents $n^{\circ}$ 138. Paris.

Sen A. (2000). Un nouveau modèle économique Développement, Justice, Liberté. Éditions Odile Jacob, Paris.

United Nations (2009). World Population Prospects. The 2008 Revision. Highlights. New York, Nations Unies, Division de la population.

Van der Ven R. and J. Smits. (20II). "The demographic window of opportunity: age structure and sub-national economic growth in developing countries ». NICE Working Paper II-I02. 